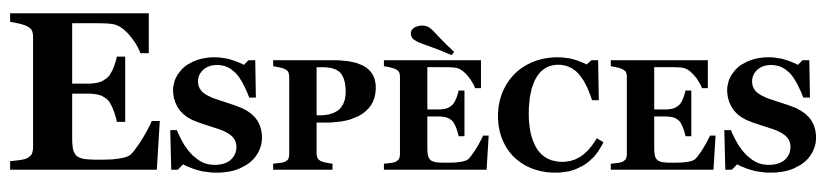

NATURELLES,
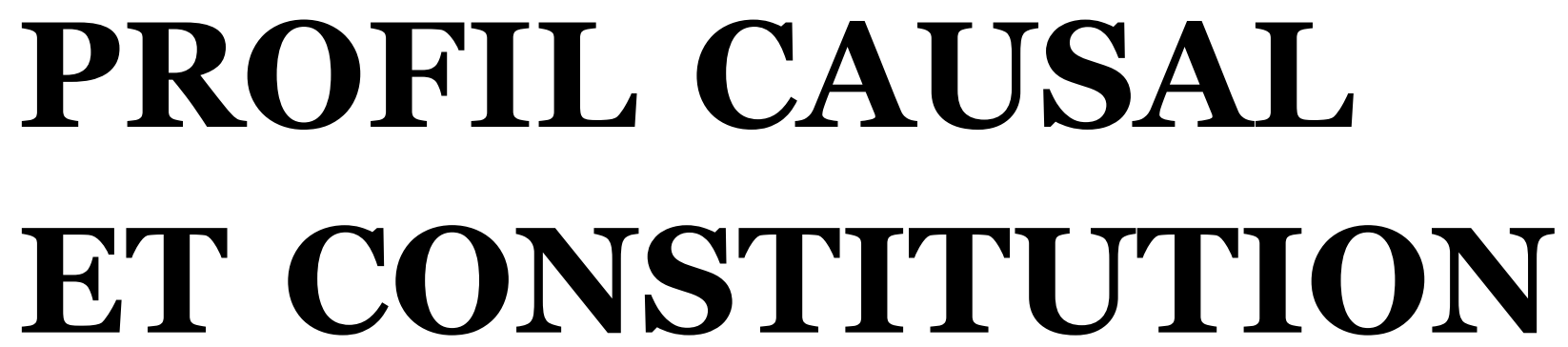

MULTIPLE

\title{
ins
}

SOCIÉTÉ DE PHILOSOPHIE DES SCIENCES (SPS) 


\section{ESPÈCES NATURELLES, PROFIL CAUSAL ET CONSTITUTION MULTIPLE}

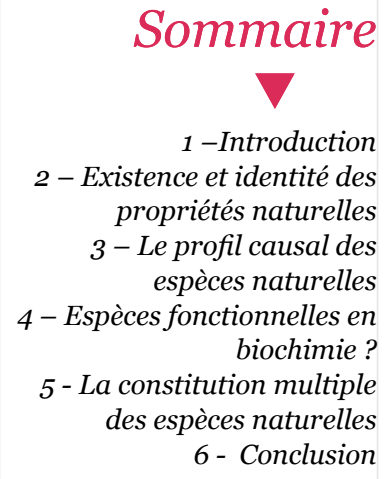

6 - Conclusion

\begin{abstract}
L'identité d'une espèce naturelle peut être conçue en termes de son profil causal. Une telle conception correspond mieux à la science que deux alternatives. L'identité d'une espèce naturelle n'est pas déterminée par un rôle causal unique parce que 1) une espèce peut avoir plusieurs rôles causaux ainsi que plusieurs fonctions et 2) certaines fonctions sont partagées par différentes espèces. Par ailleurs, en général, la thèse microstructuraliste n'est pas correcte : l'identité de certaines espèces naturelles n'est pas déterminée par leur microstructure. Il est vrai que si les substances chimiques $A$ et $B$ ont la même composition microstructurelle, alors un échantillon de l'espèce de substance $A$ appartient à la même espèce qu'un échantillon de la substance B. Cependant, l'implication inverse ne vaut pas. Le fait qu'un échantillon A appartienne à la même espèce que l'échantillon B ne garantit pas que $A$ et $B$ aient la même composition microstructurelle. Certaines espèces naturelles macroscopiques peuvent en effet être « constituées de manière multiple » ou «multiconstituées » par différentes structures microscopiques.
\end{abstract}

The identity of a natural kind can be construed in terms of its causal profile. This conception is more appropriate to science than two alternatives. The identity of a natural kind is not determined by one causal role because one natural kind can have many causal roles and several functions and because some functions are shared by different kinds. Furthermore, the microstructuralist thesis is wrong: The identity of certain natural kinds is not determined by their microstructure. It is true that if $A$ and $B$ have the same microstructural composition then a sample of a chemical substance $A$ is of the same chemical substance as a sample of $B$. However, the reverse does not hold. It is not the case that if a sample of a chemical substance $A$ is of the same chemical substance as a sample of $B$ then $A$ and $B$ have the same microstructural composition. This is because a macroscopic NK can be "multiconstituted" by different microstructures

Mots clés: espèce naturelle, profil causal, microstructuralisme, constitution, multiconstitué, fonction, rôle causal, chimie, substance.

\section{1 - Introduction}

Les espèces naturelles sont des classes d’objets, événements ou substances qui existent indépendamment des catégories humaines, conventionnelles ou non ${ }^{1}$. Il y a des cas intuitivement clairs d'espèces naturelles et d'espèces non naturelles. Le concept $\mathrm{T}$ du tremblement de terre ayant une magnitude située entre 4 et 4,9 qui eut lieu en 2014 ne correspond pas à une espèce naturelle parce que l'ensemble $\mathrm{E}$ d'événements dans l'extension de ce concept ne partage aucun aspect objectif qui est 1) indépendant du concept humain T et 2) que par ailleurs il ne partage pas avec des événements qui n'appartiennent pas à E. Une manière d'exprimer cela est de dire que : si le concept humain T n'existait pas ou s'il n'y avait pas d'humains, les événements dans E ne se ressembleraient pas davantage entre eux qu'ils ne ressemblent à des événements en dehors de E. En revanche, l'or est une espèce naturelle parce qu'il existe des aspects sous lesquels tous les atomes et échantillons d'or se ressemblent plus qu'ils ne ressemblent à d'autres objets. Cependant, même s'il n'est pas controversé que l'or soit une espèce naturelle - et E n'en est pas une - il est difficile de construire une théorie philosophique justifiant ces jugements intuitifs et fournissant un fondement pour juger des cas moins paradigmatiques (Kistler, à paraître, b). L'or est une espèce naturelle paradigmatique parce que l'appartenance à la classe des atomes et échantillons d'or est déterminée par un critère nécessaire et suffisant simple : un objet est en or si, et seulement si, il s'agit d'un atome dont le nombre atomique (à savoir le nombre de protons dans le noyau atomique) est de 79 ou s'il est composé (presque exclusivement ${ }^{2}$ ) de tels atomes. Le but de cet article est de contribuer au débat philosophique au sujet de ce que sont les espèces naturelles et de ce qui détermine leur identité, plus précisément ce qui détermine si deux objets ou deux échantillons A et B appartiennent à la même espèce.

1 - J'adopte ici la conception selon laquelle une espèce naturelle est une classe ou un ensemble d'objets plutôt qu'une entité universelle (conçue comme une substance seconde aristotélicienne), pour sa plus grande parcimonie. Pour anticiper sur un exemple qui sera mentionné dans un instant, quand on considère que l'or est une espèce naturelle, on peut interpréter cette thèse métaphysique de deux manières : selon la première - qui sera privilégiée ici - l'espèce naturelle d'or consiste en l'ensemble des atomes et échantillons macroscopiques d'or, sans distinction du lieu ou du temps de leur existence. Selon la seconde, l'or est un type universel dont les atomes et les échantillons d'or sont des instances. Ces deux conceptions correspondent aux conceptions des propriétés naturelles en tant que classes d'objets se ressemblant et en tant qu'universaux dont les objets membres de ces classes sont les instances (Bird 2015). Cette distinction entre deux interprétations de ce qu'est une espèce naturelle est indépendante des thèses et arguments examinés dans ce qui suit. 2 - Le concept d'espèce naturelle utilisé aussi bien dans le sens commun qu'en chimie admet des impuretés, ce qui rend son extension vague (Abbott 1997; Hendry 2006). 


\section{ESPÈCES NATURELLES, PROFIL CAUSAL ET CONSTITUTION MULTIPLE}

Il existe deux manières de concevoir le projet de fournir une analyse philosophique de ce que sont les espèces naturelles et de ce qui détermine leur identité. La première est de concevoir la tâche comme faisant partie de la « métaphysique descriptive » au sens de Strawson (1959). Son but est de rendre explicite la structure du concept d'espèce naturelle tel qu'il fait partie du sens commun et s'exprime dans le langage ordinaire. Une grande partie de la littérature philosophique sur les espèces naturelles appartient à cette tradition de recherche, en particulier l'analyse de la sémantique des termes d'espèces naturelles des langues naturelles. Le but d'une telle analyse est de découvrir comment le locuteur compétent d'une langue naturelle conçoit la référence ou l'extension des termes d'espèces naturelles qu'il utilise. La métaphysique descriptive ainsi interprétée a traditionnellement été conçue et poursuivie telle une philosophie « en fauteuil » (Jackson 1998) dont la méthode consiste à analyser les intuitions du philosophe lui-même. Cependant, la métaphysique descriptive peut être naturalisée par les sciences cognitives ${ }^{3}$. Par « naturalisation », on entend la tentative de soumettre un concept qui trouve son origine dans le sens commun à une analyse scientifique. Une telle entreprise de naturalisation peut aboutir à différents résultats. On peut découvrir que certaines intuitions sont déterminées - ou à tout le moins influencées - par la culture à laquelle une personne appartient ; d'autres peuvent s'avérer idiosyncrasiques au sens d'être déterminées par leur histoire personnelle. Mais, et cela semble être le cas des intuitions concernant les espèces naturelles, on peut aussi découvrir que certaines d'entre elles sont partagées par tous les êtres humains, ce qui pourrait s'expliquer par leur caractère inné. La naturalisation d'une intuition permet donc de découvrir le statut de cette dernière qui reste indéterminé dans la métaphysique de fauteuil. Les concepts d'espèce naturelle du sens commun font l'objet de recherches en psychologie cognitive (Ahn et al. 2001 ; Gelman 2003) et en anthropologie (Sousa et al. 2002). Ces recherches semblent confirmer la thèse selon laquelle le sens commun conçoit l'identité des espèces naturelles comme étant déterminée par une essence sous-jacente qui n'est en général pas directement observable. Par " essence », on entend un critère d'appartenance à une espèce dont l'application s'étend à d'autres mondes possibles, au-delà du monde actuel. Le postulat de telles essences permet d'expliquer le fait que nous avons des intuitions sur ce qui est possible même si ce n'est pas le cas dans le monde réel ${ }^{4}$.

Je n'ai mentionné cette première manière de concevoir la tâche philosophique d'une analyse du concept d'espèce naturelle que pour la distinguer d'une seconde manière selon laquelle cette tâche appartient à la métaphysique des sciences. En ce qui concerne les espèces naturelles, le but de la métaphysique des sciences est de découvrir ce que les espèces naturelles sont réellement à la lumière des informations que les sciences nous donnent à leur sujet. Les concepts scientifiques sont souvent différents des concepts découverts lors de la naturalisation du sens commun qui suppose par exemple qu'il existe quelque chose (une « essence ») que partagent tous les animaux d'une espèce donnée et qui fournit donc une condition nécessaire et suffisante à l'appartenance à cette espèce. La biologie contemporaine sur laquelle se fonde la métaphysique des espèces naturelles ne justifie pas l'idée que les animaux appartenant à une espèce partagent une propriété intrinsèque. Le concept d'espèce biologique peut néanmoins être défini sans une telle propriété intrinsèque universellement partagée, par exemple en termes de descendance commune, ce qui constitue un critère extrinsèque (Okasha 2002).

Dans la perspective de la métaphysique des sciences, l'existence d'une espèce naturelle se justifie d'une manière que je préciserai dans ce qui suit, par une ou plusieurs sciences. Certaines espèces naturelles ainsi identifiées à la lumière des théories scientifiques, mais non toutes, correspondent plus ou moins à un concept d'espèce naturelle du sens commun. Comme on vient de le voir, ces concepts du sens commun peuvent devenir eux-mêmes l'objet de recherches scientifiques en sciences cognitives et ainsi faire l'objet d'une naturalisation. Les sciences cognitives peuvent avoir pour résultat que le concept d'une certaine espèce naturelle ait lui-même le statut d'espèce naturelle cognitive, au sens d'être une entité dont l'existence est scientifiquement justifiée et partagée par tous les humains (ou une partie déterminée d'humains). Toutefois, dans tous les cas, le concept d'une espèce naturelle, même s'il s'avère être lui-même une espèce naturelle cognitive, reste bien entendu distinct de l'espèce naturelle que ce concept représente. Il a par exemple été envisagé que tous les enfants développent, à un certain stade de leur développement cognitif, le concept d'animal (Setoh et al. 2013). Les recherches psychologiques qui ont pour objet les propriétés de ce concept pourraient justifier l'hypothèse métaphysique de l'existence du concept en tant qu'espèce naturelle. Mais la question de l'existence d'un tel concept est indépendante de celle de l'existence d'une espèce naturelle qui pourrait lui correspondre, à savoir l'espèce naturelle des animaux. La première est une espèce psychologique alors que la seconde serait plutôt une espèce biologique dont l'existence ne peut être justifiée qu'à la lumière de la biologie et non des sciences cognitives.

\section{2 - Existence et identité des propriétés}

La métaphysique s'interroge sur l'existence et l'identité des types fondamentaux d'entités. Afin de développer la métaphysique à la lumière de la science, il faut trouver des critères scientifiques d'existence et d'identité. Le critère scientifique

3 - La philosophie expérimentale (Cova 2011, 2012) a un statut intermédiaire. Elle explore la structure du sens commun en analysant de manière systématique les intuitions d'un grand nombre de sujets.

4 - On parle aussi du monde « actuel » par opposition à ce qu’il aurait pu être. Dans la mesure où le présent article porte sur les espèces naturelles telles qu'elles sont conçues dans les sciences, plutôt que nos intuitions sur ces espèces naturelles, il ne sera plus question du concept d'essence dans ce qui suit. 


\section{ESPÈCES NATURELLES, PROFIL CAUSAL ET CONSTITUTION MULTIPLE}

le plus simple de l'existence d'une entité, quelle qu'en soit sa sorte - une propriété, une espèce de substance, d'événements ou d'individus - est le fait que cette entité joue un rôle dans une science.

(EX) Une entité X (propriété, espèce de substances ou d'individus) existe si, et seulement si, il existe une science qui fait référence à $\mathrm{X}$ dans les lois ou modèles qu'elle utilise pour produire des explications et des prédictions.

Ce critère est ici proposé comme une hypothèse de travail. Il est bien entendu controversé, étant donné que la possibilité d'une métaphysique des sciences fait elle-même l'objet d'une vive controverse ${ }^{5}$. Voici quelques remarques au sujet du critère $(\mathrm{EX})$ :

1. Le critère d'appartenance à une loi de la nature est plus faible que celui défendu par Quine. Selon lui, l'ontologie d'une théorie donnée consiste en l'ensemble des objets envers lesquels la théorie est " ontologiquement engagée », c'est-à-dire que les objets existent si la théorie est correcte. Techniquement, Quine propose de prendre la quantification comme critère de l'engagement ontologique et avance qu'une théorie est engagée pour l'existence d'un ensemble d'objets si, et seulement si, ces objets appartiennent au domaine dans lequel les variables liées qui apparaissent dans les axiomes et théorèmes de la théorie prennent leurs valeurs ${ }^{6}$. Pour le dire un peu plus simplement, son idée est qu'il faut examiner les généralisations universelles ( tout objet x est tel que... ») et existentielles ( il existe un objet x tel que... ») qui apparaissent dans une théorie scientifique donnée. Ce qui existe selon la théorie est l'ensemble des objets au sein duquel les variables liées de cette théorie prennent leurs valeurs. Une raison de considérer que le critère de Quine est trop fort est qu'il ne peut être appliqué à des théories dont il existe plusieurs versions formellement différentes, comme c'est notamment le cas en mécanique classique (Vorms 2011) ou encore lorsqu'il existe plusieurs interprétations, comme en mécanique quantique. Je suivrai la conception alternative proposée par Arm- strong (2004). Selon l'hypothèse dite des vérifacteurs (truthmakers), l'engagement ontologique d'une théorie scientifique s'étend également aux propriétés exprimées par les prédicats qui figurent dans les énoncés de la théorie, même si ces prédicats ne font pas l'objet de généralisations d'ordre supérieur. En ce sens, (EX) exprime l'hypothèse selon laquelle les formules de lois scientifiques nous donnent des raisons de croire en l'existence des propriétés représentées par tous les prédicats que ces formules contiennent (à l'exception des prédicats mathématiques) et non seulement en l'existence des valeurs des variables dans la portée d'un quanteur ${ }^{7}$.

2. La justification de l'existence de $X$ à partir de la présence d'un prédicat représentant $X$ dans un énoncé de loi est indépendante de la réductibilité de la loi. Il est possible que la masse soit une propriété fondamentale au sens que certaines lois portant sur la masse sont irréductibles. Mais, pour anticiper sur un exemple que je donnerai un peu plus loin, la conductivité $g_{K}$ des membranes des fibres musculaires pour les ions $\mathrm{K}^{+}$n'est certainement pas irréductible dans le sens où il est plausible qu'il existe une explication réductrice des lois portant sur $g_{K}$. Ce point est important (et mériterait d'être justifié ; $c f$. Kistler 2004, 2007, 2012, à paraître, a) dans la mesure où il existe une importante controverse concernant la portée métaphysique des " sciences spéciales », c'est-à-dire de toutes les sciences en dehors de la physique fondamentale. Selon une stratégie argumentative que l'on appelle « éliminativiste ", le fait que les hypothèses d'une théorie $\mathrm{T}$ ne soient pas réductibles, directement ou indirectement à la physique fondamentale, constitue une raison de considérer que $\mathrm{T}$ n'a pas de portée métaphysique, autrement dit que les termes théoriques de $\mathrm{T}$ ne font pas référence à des entités réelles. Paul et Patricia Churchland ont appliqué un tel argument à une partie importante de la psychologie (Churchland 1979, 1986 ; $c f$. aussi Kim 1998, Bickle 2003). D’autres ont, au contraire, fait valoir que ce qui justifie l'existence d'une science spéciale donnée $\mathrm{T}$, et donc indirectement des implications métaphysiques que l'on peut tirer de l'application du cri-

5 - Je ne peux ici rendre justice à la complexité du débat sur la possibilité de construire une métaphysique compatible avec les connaissances scientifiques actuelles. L'un des axes qui structurent la controverse est la question de savoir si la connaissance métaphysique peut - au moins en partie - être a priori, comme le soutiennent notamment Jackson (1998) et Lowe (2001), ou si elle doit être entièrement dérivée des théories scientifiques, ce qui implique que la métaphysique des sciences a le même statut de connaissance a posteriori que ces théories elles-mêmes. J'adopte la dernière conception, suivant sur ce point Ladyman et Ross (20o7), Maudlin (2007) et Callender (2011).

6 - «We may be said to countenance such and such an entity if and only if we regard the range of our variables as including such an entity. To be is to be a value of a variable. » (Quine 1939a, p. 199 ; les caractères en italique sont de Quine). Cf. aussi Quine (1939b). "We are convicted of a particular ontological presupposition if, and only if, the alleged presuppositum has to be reckoned among the entities over which our variables range in order to render one of our affirmations true " (Quine 1948, p. 13).

7 - Pour d'autres arguments en faveur de notre thèse selon laquelle nous avons autant de raisons de penser que les propriétés existent que de raisons de penser que les objets particuliers existent, cf. Mellor (1991, 2012). Mellor propose de remplacer le critère de l'engagement ontologique de Quine par ce qu'il appelle le « test de Ramsey " : si nous rassemblons toutes les lois dans un unique «énoncé de Ramsey », les propriétés qui existent (c'est-à-dire celles pour lesquelles la science est " ontologiquement engagée ») sont les valeurs des variables (de second ordre) qui sont dans la portée des quanteurs de cet énoncé de Ramsey. Cette conception conduit Mellor à nier qu'il existe des propriétés qui correspondent aux prédicats de lois non fondamentales, p. ex. chimiques, comme la propriété d'être un atome de potassium. Selon le critère de Ramsey, seules les propriétés d'être des isotopes spécifiques de potassium existent puisque son énoncé ne quantifie que sur les prédicats exprimant ces dernières et non sur le prédicat disjonctif exprimant la propriété d'être un atome de potassium de l'un ou l'autre isotope. Cf. Mellor (2012, p. 399). Cependant, Mills (2014) montre qu'il n'y a pas de raison de penser qu’il n'existe qu'un seul énoncé de Ramsey, ce qui implique que celui-ci ne soit pas suffisant pour jouer le rôle (de notre (EX)) de donner un critère nécessaire et suffisant pour être une propriété naturelle. 


\section{ESPÈCES NATURELLES, PROFIL CAUSAL ET CONSTITUTION MULTIPLE}

tère (EX) à ses hypothèses, est le fait que T ne soit pas réductible à des sciences plus fondamentales (Fodor 1974).

3. (EX) peut être justifié dans un cadre naturaliste. Le naturalisme considère que les justifications les meilleures et les plus fondamentales sont scientifiques. Dans un cadre naturaliste, la meilleure raison de croire en l'existence de $\mathrm{X}$ est le fait que la science postule $\mathrm{X}$, ce qui peut être inféré de l'usage scientifique de formules représentant des lois ${ }^{8}$ qui contiennent un prédicat représentant $\mathrm{X}$. Le fait que $\mathrm{X}$ apparaisse dans des lois reflète le fait que la science postule l'existence de $\mathrm{X}$ parce que les lois ellesmêmes jouent un rôle central dans les sciences : les lois sont notamment utilisées pour expliquer et prédire des phénomènes et pour justifier des conditionnels contrefactuels. Bien entendu, l'histoire nous donne des raisons de penser que les théories et modèles avec lesquels travaille la science présente ne sont pas définitifs, autrement dit que ces théories et modèles sont susceptibles d'être modifiés ou remplacés. L'adoption du critère (EX) a pour conséquence de rendre la métaphysique dépendante des sciences, y compris en ce qui concerne son changement au cours de l'histoire. Les entités et structures métaphysiques dont l'existence est justifiée par le critère (EX) ont un statut épistémique au moins aussi faillible que celui des théories scientifiques dont elles sont dérivées. Par ailleurs, ils sont soumis au changement historique autant qu'elles. La justification de la conception métaphysique d'une partie du monde qui s'appuie sur une théorie scientifique ne peut être plus forte que la justification de cette théorie, celle-ci étant susceptible d'être abandonnée avec elle. La métaphysique des sciences ainsi conçue est nécessairement au moins aussi provisoire, hypothétique et soumise au changement historique que les théories scientifiques elles-mêmes.

4. Selon la conception de la métaphysique des sciences fondée sur l'application du critère (EX), ses résultats dépendent des théories scientifiques auxquelles (EX) est appliqué. Ce dernier exprime l'idée que les lois et modèles jouent le même rôle à l'égard des implications métaphysiques d'une théorie. Cela peut paraître surprenant étant donné la différence qui existe entre les lois et les modèles : si les lois correspondent à des hypothèses confirmées qui ont souvent été conçues avec l'intention d'être capable d'une interprétation réaliste, de nombreux modèles sont explicitement conçus en tant que fictions incapables d'une telle interprétation réaliste (Barberousse et Ludwig 2000). Cette distinction doit être comprise dans le cadre d'une réflexion générale sur le réalisme scientifique, c'est-à-dire sur l'ambition des théories scientifiques de représenter la réalité. Toutes les hypothèses sont faillibles ; par conséquent, même celles étant bien confirmées ne peuvent être que des tentatives provisoires de représenter des entités, des propriétés ou encore des processus réels. La différence entre des structures introduites en tant que modèle - et par conséquent comme fiction - et des structures introduites dans le but de représenter des lois est une différence de degré. La plausibilité d'une théorie ou modèle, c'est-à-dire la possibilité de l'interpréter de manière réaliste, dépend de son intégration dans le reste de l'édifice scientifique. Pour ne prendre qu'un exemple, Bohr a introduit son modèle de l'atome en tant que modèle - et donc en tant que fiction - parce que ce modèle postule des entités ayant des propriétés incompatibles avec des hypothèses physiques extrêmement bien confirmées. Le modèle de Bohr postule par exemple que les atomes contiennent des électrons qui peuvent se trouver sur des orbites stationnaires fermés sans émettre de rayonnement. De tels électrons, ou de tels orbites, sont introduits comme fiction puisque les autres hypothèses confirmées de la physique interdisent qu'ils soient interprétés de manière réaliste. À cause de cette incompatibilité, le modèle de Bohr n'a été considéré que comme une étape en vue d'élaborer un édifice théorique plus cohérent et donc plus satisfaisant. De manière générale, l'existence de contradictions entre différentes théories et l'existence de phénomènes qu'aucune théorie n'explique, renforce le statut hypothétique et faillible des structures en vertu desquelles les théories sont ontologiquement engagées et qui font l'objet de la métaphysique des sciences.

J'utiliserai (EX) pour trouver un principe qui rend explicite la manière dont l'identité d'une propriété naturelle $\mathrm{X}$ est déterminée à la lumière de la science. Je postulerai ensuite que l'on peut obtenir une conception des espèces naturelles, adéquate à la science, en la généralisant à partir du concept de propriété naturelle. Dans les sciences avancées qui ont recours à un formalisme mathématique et représentent les généralisations nomologiques sous forme d'équations, les symboles qui ne représentent pas des nombres ou des relations logiques ou mathématiques représentent typiquement des propriétés naturelles. Selon (EX), l'existence de la propriété naturelle d'avoir une masse déterminée (ainsi que de la propriété déterminable d'avoir une masse, en d'autres termes d'être massif) se justifie par la présence du symbole « $\mathrm{m}$ » qui apparaît dans des équations représentant des lois de la nature telle que :

$$
W \mathrm{p}=m g h
$$

Cette loi spécifie que l'énergie potentielle $W$ p qu'un corps de masse $m$ acquiert lorsqu'il est soulevé jusqu'à une hauteur $h$ en partant de la surface de la Terre où « $g$ » représente l'accélération de la chute libre d'objets massifs qui se trouvent près de la surface de la Terre. 


\section{ESPÈCES NATURELLES, PROFIL CAUSAL ET CONSTITUTION MULTIPLE}

Voici un second exemple de propriété naturelle, beaucoup plus spécifique, dont l'existence peut être justifiée dans le cadre d'une discussion métaphysique par (EX). Les membranes cellulaires des fibres musculaires présentent une certaine conductivité pour les ions de potassium $\mathrm{K}^{+}$; en d'autres termes, ces membranes sont perméables aux ions $\mathrm{K}^{+}$dans certaines conditions et dans une certaine mesure. L'usage du critère (EX) justifie la croyance en l'existence d'une propriété naturelle $\mathrm{g}_{\mathrm{K}}$ des membranes cellulaires des fibres musculaires d'avoir une certaine conductivité pour les ions $\mathrm{K}^{+}$parce que les lois de la biophysique (Morris et Lecar 1981) contiennent un symbole $g_{K}$ représentant cette propriété. C'est le cas de la loi suivante :

$$
I=C V^{\prime}+g_{L}\left(V_{\mathrm{L}}\right)+g_{C a} M\left(V-V_{C a}\right)+g_{K} N\left(V-V_{K}\right)
$$

qui contient les symboles suivants représentant les propriétés correspondantes :

$I$ = courant électrique appliqué $\left(\mathrm{mA} / \mathrm{cm}^{2}\right)$

$C$ = capacité de la membrane $\left(\mathrm{mF} / \mathrm{cm}^{2}\right)$

$V$ = potentiel de la membrane, $V_{L}, V_{C a}, V_{K}=$ potentiels d'équilibre correspondant au conductivités $g_{L}, g_{C a}, g_{K}$

$V^{\prime}=$ dérivée du potentiel par rapport au temps, représentant son taux de variation

$g_{L}, g_{C a}, g_{K}=$ conductivité résiduelle, conductivité pour ions $\mathrm{Ca}^{++}$et pour ions $\mathrm{K}^{+}\left(\mathrm{mSiemens} / \mathrm{cm}^{2}\right)$

$M, N=$ fraction des canaux ouverts spécifiques aux ions $\mathrm{Ca}^{++}$ et $\mathrm{K}^{+}$.

Cette loi exprime le fait que si l'on applique un courant d'intensité $I$ à la membrane, ce courant se divise en quatre composantes : une partie proportionnelle à la capacité $C$ de la membrane est stockée sous forme d'électrons dans la membrane. Les trois autres parties correspondent à des charges qui traversent la membrane sous forme d'ions : une fraction proportionnelle au nombre de canaux ioniques perméables aux ions calcium $\left(\mathrm{Ca}^{++}\right)$et à la conductivité de la membrane à ces ions $\mathrm{Ca}^{++}$correspond au flux d'ions $\mathrm{Ca}^{++}$à travers ces canaux, une autre composante correspond au flux d'ions $\mathrm{K}^{+}$qui traverse la membrane par des canaux ioniques spécifiques aux ions $\mathrm{K}^{+}$et une troisième au flux net d'autres ions à travers d'autres canaux ioniques.

La stratégie naturaliste de trouver un critère d'existence des propriétés naturelles peut aussi être employée pour trouver un critère d'identité des propriétés naturelles, en d'autres termes un critère qui détermine à quelles conditions deux symboles représentent la même propriété. Quelle est la jus- tification scientifique qui permet d'évaluer si les propriétés $F$ et $\mathrm{G}$ sont différentes ? Pour montrer que $\mathrm{F} \neq \mathrm{G}$, il est nécessaire et suffisant de trouver au moins une situation dans laquelle un objet ayant $\mathrm{G}$ se comporte différemment d'un objet ayant F. Cependant, si l'on présuppose le déterminisme, cela requiert qu'il y ait au moins une loi qui contienne $\mathrm{F}$ mais non $\mathrm{G}$ (ou l'inverse) ou une loi dans laquelle $\mathrm{F}$ occupe un rôle structurel différent du rôle que joue $\mathrm{G}$ dans les lois. (S’il existe des lois statistiques, alors pour montrer que $\mathrm{F} \neq \mathrm{G}$, il est nécessaire et suffisant de trouver au moins un type de situation dans laquelle le comportement des objets ayant $\mathrm{G}$ est statistiquement différent du comportement des objets ayant F.) Autrement dit, F figure dans les mêmes lois que $G$ en $y$ occupant le même rôle structurel si, et seulement si, F = G. Ce raisonnement suggère le critère naturaliste suivant de l'identité des propriétés naturelles.

(ID) L'identité d'une propriété $\mathrm{P}$ est déterminée par l'ensemble des lois qui contiennent $\mathrm{P}$ et le rôle structurel que $\mathrm{P}$ occupe dans ces lois.

En d'autres termes, les lois qui contiennent une propriété $\mathrm{P}$ font de la propriété $P$ ce qu'elle est. Cette thèse métaphysique doit être distinguée de la thèse épistémique selon laquelle une propriété $\mathrm{P}$ est connue dans la mesure où les lois qui la contiennent sont connues. Selon la thèse métaphysique (Shoemaker 1980 ; Kistler 2002), les lois qui contiennent P déterminent l'identité de $\mathrm{P}^{9}$.

Les propriétés naturelles postulées dans les sciences sont toujours présentes dans plus d'une seule loi. Voici trois lois qui contiennent la charge électrique élémentaire q.

1. $\quad \vec{F}=q \vec{E}$. Un objet portant la charge électrique élémentaire q qui est situé à un point de l'espace où la force du champ électrique est $\vec{E}$ subit une force $\vec{F}$ dont la force et la direction est $q \vec{E}$.

2. $\vec{F}=q \vec{v} \times \vec{B}$. Selon la loi de la force de Lorentz, un objet qui porte la charge électrique élémentaire $q$ et qui traverse avec une vitesse $\vec{v}$ un champ magnétique $\vec{B}$ subit une force dont la quantité et la direction sont donnés par $q \vec{v} \times \vec{B}$.

3. $\vec{\mu}=\frac{1}{2} q \vec{r} \times \vec{v}$. Un objet chargé avec $q$, en rotation avec une vitesse $\vec{v}$ autour d'un cercle de rayon $\vec{r}$, est source d'un moment magnétique $\frac{1}{2} q \vec{r} \times \vec{v}$.

Chaque loi qui contient la charge élémentaire $q$ détermine une disposition partagée par tous les objets qui portent $q$. Un prédicat dispositionnel peut être caractérisé par un condi-

9 - Cette thèse a des conséquences modales. Dans chaque monde possible dans lequel P existe, toutes les lois qui contiennent P existent. Si une propriété $P^{*}$ ne figure pas dans l'une des lois portant sur $P$ ou n'y figure pas de la même manière, alors $P^{*}$ n'est pas identique à $P$. Une propriété qui fait qu'un objet qui la possède attire (ou repousse) d'autres charges avec une force proportionnelle à $1 / r^{3}$ plutôt qu'avec une force proportionnelle à $1 / r^{2} n^{\prime}$ 'est pas la charge électrique. En d'autres termes, une telle propriété n'est pas la charge électrique existant dans le monde réel (autrement dit, le monde " actuel » au sens modal), parce que l'identité de la propriété d'être chargé électriquement est déterminée, entre autres choses par le fait que les objets chargés attirent (ou repoussent) d'autres objets chargés avec une force proportionnelle à $1 / r^{2}$. Lorsqu'on envisage une propriété $P^{*}$ comme on vient de le faire, il s'agit d'une possibilité métaphysique : une propriété qui existe dans un monde possible où il existe une force proportionnelle à $1 / r^{3}$. Le statut de telles possibilités métaphysiques est controversé : pour Lewis (1986), tous les mondes possibles ont le même statut de réalité que notre monde que l'on appelle le monde « actuel », alors que pour d'autres, comme Armstrong (1989), les mondes possibles (non actuels) ont le statut de fictions. 


\section{ESPÈCES NATURELLES, PROFIL CAUSAL ET CONSTITUTION MULTIPLE}

tionnel contrefactuel $\mathrm{T} \square \rightarrow \mathrm{M}$, où $\square \rightarrow$ représente le connecteur contrefactuel, $\mathrm{T}$ la condition de déclenchement caractéristique de la disposition et $\mathrm{M}$ la manifestation caractéristique de la disposition relativement à T. En vertu de la loi $\vec{F}=q \vec{E}$ les objets chargés avec $q$ ont la disposition suivante : si un objet chargé de charge $q$ était dans $\vec{E}$, il subirait une force $q \vec{E}$ (T : être dans $\vec{E} ; \mathrm{M}:$ subir une force $q \vec{E}$ ). En vertu de la loi $\vec{F}=q \vec{v} \times \vec{B}$, des objets chargés de charge $q$ ont la disposition suivante : si un objet chargé avec $q$ se mouvait avec une vitesse $\vec{v}$ à travers un champ magnétique $\vec{B}$, il subirait une force de Lorentz égale à $q \vec{v} \times \vec{B}$.

La présence de $q$ dans ces lois suffit selon (EX) pour postuler l'existence de la propriété de porter la charge électrique élémentaire. Chacune de ces lois est à l'origine d'une disposition que les objets ont en vertu de leur possession de la charge élémentaire $q$. De la prémisse selon laquelle toutes les lois comportant $q$ sont essentielles à $q$ (au sens où q ne pourrait pas exister sans ces lois) et de la prémisse selon laquelle chacune de ces lois est à l'origine d'une disposition des objets possédant la charge élémentaire, il découle que toutes ces dispositions sont essentielles à la propriété d'avoir la charge élémentaire $q$, au sens où rien ne pourrait posséder la propriété sans avoir les dispositions. La possession de la propriété d'avoir $q$ est nécessaire et suffisante pour avoir toutes ces dispositions. $\mathrm{Si}$ les lois et les dispositions sont indissociables et si les lois portant sur une propriété $\mathrm{P}$ déterminent ce qu'est $\mathrm{P}$, alors on aboutit à la théorie dispositionnelle de l'identité des propriétés naturelles (Shoemaker 1980) selon laquelle l'identité d'une propriété $\mathrm{P}$ est déterminée par l'ensemble des dispositions que $\mathrm{P}$ donne aux objets qui possèdent $\mathrm{P}$, en d'autres termes par son profil causal.

Si la propriété $\mathrm{P}$ est indissociable de l'ensemble des lois dans lesquelles elle figure et si chaque loi est inséparable d'une disposition, alors les dispositions correspondant à la possession de $\mathrm{P}$ sont également inséparables entre elles, au sens où un objet possède l'une de ces dispositions si, et seulement si, ce dernier les possède toutes. Si la solidarité de certains ensembles de dispositions est une conséquence de l'existence d'une propriété naturelle, comme dans les thèses métaphysiques mentionnées, cette solidarité, qui est un fait constaté par les sciences, peut aussi être utilisée en tant que prémisse dans une inférence à la meilleure explication visant à justifier l'existence des propriétés naturelles. L'existence d'une propriété naturelle $\mathrm{P}$, présente dans plusieurs lois, fournit une explication de l'association systématique d'un ensemble de dispositions. Les trois dispositions qui correspondent aux trois lois mentionnées plus haut sont systématiquement associées : aucun objet ne présente qu'une seule - voire même deux - de ces dispositions. Le postulat d'une propriété naturelle q sous-jacente aux trois dispositions explique le fait que ces trois dispositions sont inséparables, ce qui resterait autrement un « fait brut » sans explication.
La propriété naturelle est le fondement commun de toutes ces dispositions. Ce fondement est ce que Prior, Pargetter et Jackson (1982) appellent « la base causale » : l'ensemble des propriétés intrinsèques qui contribuent, avec la condition déclenchante de la disposition, à produire causalement la manifestation. Selon cette conception des propriétés naturelles, elles sont des dispositions « multi-track » ou dispositions à manifestations multiples (Kistler 2012). Il s'agit de propriétés qui se manifestent de différentes manières et dans différentes circonstances. Bird (2007) soutient que de telles propriétés ne peuvent pas être fondamentales. Dans le vocabulaire de Bird, une disposition est appelée " pure » si elle ne se manifeste que d'une seule manière. L'objet $b$ a la disposition pure $\mathrm{D}$ si la possession de $\mathrm{D}$ ne donne lieu qu'à une seule disposition de manifester $\mathrm{M}$ dans des circonstances de déclenchement $\mathrm{T}$ :

$$
\text { (Db et } \mathrm{Tb}) \square \rightarrow \mathrm{Mb} \text {. }
$$

Selon Bird, « il n'est pas nécessaire de postuler des dispositions fondamentales multi-track » (Bird 2007, p. 24) dont la possession donne lieu à tout un ensemble de conditionnels :

$$
\forall \mathrm{i}\left[\left(\mathrm{Ib} \text { et } \mathrm{T}_{\mathrm{i}} \mathrm{b}\right) \square \rightarrow \mathrm{M}_{\mathrm{i}} \mathrm{b}\right]
$$

parce que toutes les dispositions impures sont équivalentes à des conjonctions de disposition pures (« single-track») (Bird 2007, p. 23).

Cet argument appelle deux remarques :

1. Le fait qu'une propriété apparaisse dans plus d'une loi garantit qu'elle donne à ses possesseurs plus d'une disposition. Par conséquent, l'existence de dispositions pures au sens de Bird est douteuse : une propriété ne peut procurer une telle disposition pure que si elle n'apparaît que dans une seule loi. Mais le postulat d'une telle propriété serait superflu. Si m ne figurait que dans la loi $\mathrm{F}=\mathrm{ma}$, nous pourrions considérer que $\mathrm{m}$ soit défini par F/a. Il est essentiel, pour justifier le postulat d'une propriété théorique, que son identité ne soit pas exhaustivement spécifiée par une seule loi prise en isolation dans laquelle elle figure (Hempel 1965).

2. Une propriété naturelle théorique ${ }^{10}$ telle que la charge électrique ne se réduit pas à la conjonction de ses dispositions caractéristiques (une pour chaque loi connue dans laquelle elle figure). Une propriété théorique fournit une explication unificatrice de cet ensemble de dispositions. Elle explique pourquoi les dispositions sont dans l'ensemble inséparables les unes des autres. Le postulat qu'il existe une propriété qui correspond à la variable « $\mathrm{m}$ » présente dans différentes lois explique pourquoi les dispositions qui correspondent à ces lois dans lesquelles « $\mathrm{m}$ » apparaît sont indissociables. C'est parce

10 - Le raisonnement exposé s'appuie sur les lois dans lesquelles figure une propriété donnée. Il ne s'applique donc qu'aux propriétés qui figurent dans de telles lois, autrement dit qui font l'objet de théories scientifiques. On peut bien entendu spéculer que la conception justifiée ainsi s'applique également à d'autres propriétés qui ne font pas (encore) l'objet de théories scientifiques. Mais il est prudent de limiter la portée de l'argument aux propriétés théoriques. 


\section{ESPÈCES NATURELLES, PROFIL CAUSAL ET CONSTITUTION MULTIPLE}

que « $\mathrm{m}$ » correspond à une seule propriété qu'un objet peut avoir - ou ne pas avoir - mais qu'il est impossible de n'avoir que « partiellement », qu'il est impossible que son comportement n'obéisse qu'à une partie des lois qui contiennent « $\mathrm{m}$ », sans obéir à toutes.

\section{3 - Le profil causal des espèces naturelles}

Il semble prometteur de concevoir la catégorie métaphysique des espèces naturelles en analogie avec la catégorie des propriétés naturelles. Une espèce naturelle d'objets tels que les électrons, les ions $\mathrm{K}^{+}$ou les canaux ioniques perméables aux ions $\mathrm{K}^{+}$dans la membrane des fibres musculaires, est un ensemble d'objets ou d'échantillons de substances qui se ressemblent par certains aspects, en d'autres termes qui partagent certaines propriétés naturelles, plus qu'ils ne ressemblent aux objets et substances en dehors de l'ensemble ${ }^{11}$. Dans le cas d'espèces fondamentales telles que les électrons, les membres de l'espèce se ressemblent parfaitement, c'està-dire qu'ils partagent toutes leurs propriétés intrinsèques. Pour les propriétés non fondamentales, la ressemblance n'est en général pas parfaite. Les ions $\mathrm{K}^{+}$peuvent appartenir à différents isotopes tels que le ${ }^{39} \mathrm{~K}$ et le ${ }^{40} \mathrm{~K}$. Les ions $\mathrm{K}^{+}$de différents isotopes appartiennent à la même espèce naturelle en vertu du fait qu'ils partagent le même nombre de protons dans leur noyau ainsi que leur configuration électronique. Toutefois, ils diffèrent à l'égard du nombre de neutrons : le ${ }^{39} \mathrm{~K}$ et le ${ }^{40} \mathrm{~K}$ possèdent tous les deux 19 protons mais les atomes de l'isotope ${ }^{39} \mathrm{~K}$ possèdent 20 neutrons alors que ceux de l'isotope ${ }^{40} \mathrm{~K}$ en possèdent 21. Le fait que ce qui détermine l'appartenance à l'espèce naturelle $\mathrm{K}$ du potassium soit le nombre atomique (le nombre de protons) et non la masse (elle-même déterminée par la somme des protons et des neutrons) s'explique par le fait qu'il s'agit ici d'une espèce naturelle chimique. Or, du point de vue de la chimie, elle-même fondée sur la physique atomique, le comportement des atomes, notamment en ce qui concerne leur réactivité et leur capacité à former des molécules, est déterminé par leur structure électronique, celle-ci étant déterminée par le nombre d'électrons lui-même déterminé par le nombre de protons dans le noyau de l'atome électriquement neutre.

Dans les deux cas (celui des espèces fondamentales et celui des espèces non fondamentales), une espèce naturelle peut être caractérisée par un ensemble de propriétés systématiquement associées entre elles. Un tel ensemble de propriétés constitue ce que Boyd (1991) appelle un « cluster homéostatique de propriétés » (homeostatic property cluster : HPC) et Slater (2015), un « cluster stable de propriétés » (stable property cluster : SPC). Les propriétés naturelles sont « systématiquement 'sociables' de différentes manières. Les unes aiment être accompagnées des autres.” (Chakravartty 2007, p. 170$)^{12}$

Les explications qui font intervenir les espèces naturelles exploitent cette association systématique. Les explications biochimiques qui font référence aux ions $\mathrm{K}^{+}$dépendent du fait que ces derniers présentent plusieurs propriétés. La charge positive des ions $\mathrm{K}^{+}$ne suffit pas à elle seule à expliquer la contribution des ions $\mathrm{K}^{+}$à un mécanisme tel que la propagation du potentiel d'action. Pour preuve, les ions $\mathrm{Na}^{+}$, qui sont également chargés positivement, jouent un rôle complètement différent dans le même mécanisme. Les protéines, fondamentales pour le fonctionnement des mécanismes biochimiques, jouent leur rôle en vertu d'un très grand nombre de propriétés : non seulement la charge électrique nette et la masse, mais encore la distribution des charges et la conformation spatiale sont indispensables à leur contribution à ces mécanismes.

D’après ce qui précède, il pourrait sembler qu'il ne soit pas nécessaire de postuler l'existence d'une espèce naturelle au-delà de l'ensemble des propriétés partagées par tous les membres de l'espèce. On peut songer à la thèse de Bird selon laquelle une propriété qui donne à ses possesseurs de nombreuses dispositions peut être analysée en termes de conjonction de propriétés correspondant chacune à une disposition simple ou « pure ». Par un raisonnement analogue, des auteurs tels qu'Armstrong (1997), Bird (2007) et Tobin (2013) parviennent à l'idée qu'une espèce naturelle peut être analysée comme équivalente à un ensemble de 1) plusieurs propriétés et 2) de lois et mécanismes qui assurent la coexistence de ces propriétés. La parcimonie exige de s'abstenir de postuler des entités qui ne sont pas strictement indispensables pour l'interprétation de la science. "Les espèces naturelles sont une addition ontologique superflue. Une ontologie qui ne postule que les propriétés suffira pour déterminer les classifications naturelles » (Tobin 2013, p. 175). S'il est en principe possible de fournir une explication réductrice de la coexistence systématique de l'ensemble de propriétés caractéristiques d'une espèce donnée, pourquoi postuler l'existence de l'espèce naturelle en tant qu'entité qui existerait en plus des propriétés, lois et mécanismes mentionnés dans l'explication réductrice ? Il peut sembler superflu de postuler l'existence de l'espèce naturelle des ions $\mathrm{K}^{+}$, outre les propriétés d'être un atome dont le noyau contient 19 protons et la configuration électronique caractéristique d'un atome de 18 électrons. Une fois l'explication réductrice connue, les propositions scientifiques portant sur les ions $\mathrm{K}^{+}$peuvent être en principe éliminées et remplacées par des propositions dans lesquelles ne figure plus qu'une description structurelle faisant

11 - De manière analogue aux propriétés naturelles, il existe deux manières de concevoir les espèces naturelles comme des classes d’objets ou comme des universaux.

12 - Les propriétés naturelles peuvent être sociables « de différentes manières » au sens où certaines espèces naturelles (que Chakravartty appelle « essence kinds ») - mais non toutes - sont caractérisées par le fait que leurs membres partagent toutes leurs propriétés intrinsèques. Les électrons sont une telle espèce " essentielle » dans la mesure où ils partagent toutes leurs propriétés intrinsèques telles que leur masse de repos, leur charge électrique et leur spin. Dans le cas d'autres espèces (que Chakravartty appelle « cluster kinds ») seule une partie des propriétés est partagée par l'ensemble des membres de l'espèce. Tous les atomes K de potassium ont 19 protons dans leur noyau mais certains ont 20 neutrons alors que d'autres en ont 21. 
référence aux propriétés qui constituent la base de réduction, ainsi qu'aux lois et mécanismes qui assurent leur coexistence systématique.

Pour évaluer cette hypothèse, il faut d'abord établir une distinction entre lois et mécanismes. En règle générale, les espèces naturelles ne sont pas équivalentes à des ensembles de propriétés dont l'association systématique s'explique par un mécanisme : les espèces naturelles fondamentales d'objets ne peuvent pas être analysées en termes de mécanismes parce que ces objets n'ont pas de parties et ne sont donc pas complexes. Prenons les électrons. La masse, la charge et le spin caractéristiques des électrons sont indissociablement associées et forment donc ce que l'on peut appeler avec Slater (2015), un cluster stable. Cependant, étant donné que les électrons sont une espèce fondamentale, la coexistence de cet ensemble de propriétés ne peut pas être expliquée de manière mécaniste. Une explication mécaniste requiert un objet ou un système complexe : il est essentiel pour une explication mécaniste de faire référence aux parties du système dont il s'agit d'expliquer les propriétés et le comportement, ainsi qu'aux interactions entre ces parties. Les espèces naturelles fondamentales, telles que les électrons, n'ont pas de telles parties. Par conséquent, il ne peut y avoir d'explication mécaniste du fait que la charge, la masse et le spin de l'électron soient inséparables. Le fait de postuler l'existence de l'électron en tant qu'espèce naturelle constitue un cadre métaphysique pour l'expliquer. Ce cadre peut ensuite être complété par une explication scientifique.

Cependant, rien ne semble s'opposer à la possibilité que les espèces naturelles fondamentales soient réductibles à l'ensemble de leurs propriétés constitutives avec les lois qui assurent leur association ${ }^{13}$. Dans de nombreux cas, nous ignorons comment opérer une telle réduction. Dans cette situation, l'analyse suivante s'impose. Si toutes les espèces naturelles sont en principe réductibles, le concept d'espèce naturelle n'est pas métaphysiquement primitif. Cependant, dans la mesure où nous ignorons la réduction complète des espèces naturelles fondamentales, le concept d'espèce naturelle demeure épistémiquement indispensable. Dans la mesure où notre connaissance des propriétés et lois constitutives d'une espèce naturelle est partielle ${ }^{14}$, l'ensemble des propriétés qui sont connues à un moment donné de l'histoire ne constitue pas une condition nécessaire et suffisante pour l'appartenance à cette espèce.

\section{4 - Espèces fonctionnelles en biochimie?}

Si l'identité d'une espèce naturelle est déterminée par un ensemble de propriétés naturelles structurées en vertu de lois et si l'identité des propriétés naturelles est déterminée par un ensemble de dispositions, autrement dit en termes d'un profil causal, l'identité d'une espèce naturelle est elle aussi déterminée par un profil causal. En ce qui concerne les espèces naturelles qui jouent un rôle en biologie, et en particulier les espèces biochimiques, cette conclusion semble justifier la thèse selon laquelle l'identité des espèces naturelles biochimiques est déterminée en termes de ce qui remplit un rôle fonctionnel (Manafu 2014, et article non publié). Dans la suite de cet article, nous examinerons cette thèse. La relation entre les espèces et les rôles fonctionnels est complexe, pour deux raisons complémentaires : la première est qu'une espèce naturelle peut en principe avoir de nombreuses fonctions, la seconde étant que différentes espèces peuvent en principe partager la même fonction.

Les protéines que l'on appelle les «protéines de clair de lune » (« moonlighting proteins ») appartiennent à la première catégorie. Leur nom rappelle les personnes qui occupent un deuxième emploi auquel elles se consacrent de nuit. Les cristallines appartiennent à cette catégorie de molécules biologiques. Dans le cristallin des yeux des vertébrés, ces molécules remplissent la fonction structurelle qui est de donner au cristallin de l'œil la transparence et les propriétés réfractives fonctionnelles. Mais ces mêmes protéines remplissent aussi des fonctions très différentes. La cristalline $\alpha B$ a, outre sa fonction de protéine structurelle du cristallin des yeux, la fonction de protéger les cellules contre les températures élevées. La cristalline $\alpha \mathrm{B}$ sert de « petite protéine de choc thermique » (De Jong, Leunissen et Voorter 1993) dont la synthèse est déclenchée par la chaleur et d'autres formes de stress physiologique. En outre, les cristallines jouent le rôle de «protéines chaperon » qui agissent en tant qu'enzymes à différentes étapes de l'assemblage des protéines complexes (Saibil 2013).

Le fait que les protéines cristallines remplissent différentes fonctions dans différentes circonstances ne remet pas leur existence en cause, bien au contraire. Ce fait constitue en effet la meilleure justification de leur existence en tant qu'espèce naturelle. Si une molécule avait une seule fonction, il serait plus parcimonieux de postuler simplement une propriété dispositionnelle. Si la molécule avait un nombre bien déterminé de fonctions, on pourrait éviter de postuler une

13 - Cela vaut à plus forte raison pour les espèces non fondamentales, c'est-à-dire des types d'objets ou de substances qui sont composés d'objets ou de substances plus fondamentales. Les ions $\mathrm{K}^{+}$sont composés de protons, neutrons et électrons. Dans la mesure où la composition est connue, il est en principe possible de remplacer les expressions faisant référence aux ions $\mathrm{K}^{+}$par la description structurelle complète qui correspond à la réduction des ions $\mathrm{K}^{+}$à leurs composantes et aux relations entre ces composantes.

14 - La recherche scientifique au sujet de l'eau lïllustre bien. Bien que la structure de l'eau soit simple comparativement à d'autres espèces non fondamentales telles que les molécules organiques, de nouvelles propriétés de l'eau sont régulièrement découvertes. Cf. Malaterre (2010, chap. 6 et 7). En ce sens, Mill explique qüil est essentiel au concept d'espèce naturelle d'avoir plus de propriétés que celles à l'aide desquelles nous déterminons la référence du terme que nous utilisons pour la désigner: les espèces réelles sont des « classes, distinguished by unknown multitudes of properties, and not solely by a few determinate ones » (Mill 1843, I, 7, § 4, p. 80). 


\section{ESPÈCES NATURELLES, PROFIL CAUSAL ET CONSTITUTION MULTIPLE}

espèce en faisant simplement référence à un ensemble bien déterminé de propriétés dispositionnelles. Cependant, pour la même raison que dans le cas des propriétés naturelles, le postulat d'une espèce naturelle sous-jacente à différentes fonctions offre un cadre métaphysique pour une explication unificatrice du fait que les dispositions qui correspondent à ces différentes fonctions sont systématiquement associées. En outre, le nombre de fonctions d'une espèce naturelle biologique donnée n'est pas fixé à un moment donné. Dans le processus de l'évolution, la même protéine peut acquérir de nouvelles fonctions et en perdre d'autres. Pour pouvoir rendre compte de la possibilité qu'une protéine acquiert de nouvelles fonctions, il faut postuler une entité logiquement indépendante de tout ensemble fini de fonctions.

Cela suggère une conception des espèces naturelles qui en fait des entités douées de pouvoirs ${ }^{15}$ : tous les objets qui appartiennent à une espèce naturelle possèdent un certain nombre de dispositions à évoluer et interagir d'une certaine manière dans des circonstances déterminées. Certaines de ces dispositions correspondent à des fonctions biologiques. À un moment donné, seule une partie de ces dispositions se manifeste et nous n'en connaissons qu'une partie. L'espèce naturelle est sous-jacente à cet ensemble de dispositions et le détermine. Chaque fonction qu'une molécule biologique exerce peut être interprétée comme une manifestation correspondant à l'une de ces dispositions.

Différents mécanismes permettent aux protéines de clair de lune d'exercer différentes fonctions dans différentes circonstances. Cependant, dans chacun de ces mécanismes, « un changement dans l'environnement moléculaire » (Tompa , Szasz et Buday 2005, p. 484) est « responsable du fait que la protéine exerce différentes fonctions dans différentes situations » (Goodwin 2011, p. 542). Dans le cadre métaphysique des pouvoirs et des dispositions, une molécule de protéine peut être interprétée comme un objet doué d'un pouvoir $\mathrm{P}$ tel que, pour chaque condition déclenchante $\mathrm{T}_{\mathrm{i}}, \mathrm{P}$ et $\mathrm{T}_{\mathrm{i}}$ provoquent ensemble un comportement fonctionnel spécifique $\mathrm{M}_{\mathrm{i}}$. Comme les propriétés naturelles sont « multi-track » au sens où elles déterminent différentes dispositions de leurs possesseurs (une pour chaque loi contenant la propriété ; voir plus haut), les espèces naturelles sont « multi-track » au sens où elles déterminent tout un ensemble de fonctions correspondant à des manifestations différentes dans des circonstances différentes. Le fait que le pouvoir $\mathrm{P}$ détermine tout un ensemble de dispositions (la relation entre $\mathrm{P}$ et les dispositions étant donc "many-one ») explique pourquoi, par exemple, « il n'y a pas de corrélation une à une entre la structure tertiaire d'une protéine et sa fonction biologique » (Goodwin 2011, p. 542).

Le profil multifonctionnel d'une espèce naturelle montre que son identité n'est pas déterminée par une fonction singulière. Elle n'est pas non plus déterminée par l'ensemble de dispositions ou de fonctions, actualisé ou connu à un moment donné de l'histoire. Cependant il serait erroné d'en conclure que les espèces naturelles ne sont pas des « espèces fonctionnelles ». Le fait qu'une espèce ait différentes dispositions et fonctions suggère plutôt de concevoir les espèces naturelles comme ce qui est sous-jacent à un ensemble indissociable de dispositions et le détermine, certaines de ces fonctions correspondant à des fonctions biologiques. L'ensemble de ces dispositions constitue le profil causal de l'espèce naturelle, la partie connue de ce profil constituant notre base inductive pour postuler son existence. Néanmoins, l'espèce naturelle elle-même n'est pas logiquement équivalente à l'ensemble de fonctions exécutées à un moment donné de l'histoire, parce qu'il est essentiel, au moins dans le cas des protéines, qu'elle puisse acquérir de nouvelles fonctions ${ }^{16}$.

Il y a une deuxième raison complémentaire pour laquelle il n'existe pas de relation une à une entre les molécules biologiques et les fonctions. La première est le fait qu'un type donné de molécules peut remplir plusieurs fonctions, la seconde que différentes molécules peuvent partager une fonction. Il y a de nombreux exemples de fonctions biologiques partagées. En termes d'évolution, cela s'explique par « l'évolution convergente », ou plus précisément par la « convergence fonctionnelle »(Buller et Townsend 2013). Les molécules transportant l'oxygène offrent un cas exemplaire. Au moins trois types de molécules ont évolué pour transporter l'oxygène dans le système circulatoire vers les différents tissus dans le corps des animaux ${ }^{17}$. Les hémoglobines représentent le type le mieux connu assurant cette fonction dans les vertébrés et dans différents phyla (ou embranchements) d'invertébrés. Dans d'autres embranchements d'invertébrés, la fonction de transport d'oxygène est assurée par des molécules de deux autres types : hémérythrines et hémocyanines. Chacun de ces types inclut une très grande variété de molécules différentes (van Holde et Miller 1995).

15 - La conception des espèces naturelles comme entités dotées de pouvoirs ressemble à l'analyse proposée par Dumsday (2013) qui explique lui aussi que les espèces naturelles permettent d'expliquer le fait que certains ensembles de propriétés sont indissociables les unes des autres. "The natural kind essence of an object is irreducible to the properties associated with that essence, such that the kind 'electron' is something over and above its mass, charge etc., and serves to ground them. » (Dumsday 2013, p. 874). Cependant, Dumsday met l'accent sur l'argument métaphysique en faveur de l'existence d'une telle " essence », ce qui suggère lidée erronée selon laquelle les espèces naturelles doivent être postulées par les métaphysiciens plutôt que par les scientifiques. La science elle-même postule les espèces naturelles parce que leur existence permet de donner une explication théorique et empiriquement justifiable du fait que certaines dispositions sont indissociables les unes des autres.

16-Il me semble qülly a deux erreurs dans l'analyse des protéines de clair de lune par Tobin (2010). Premièrement, il est erroné de tirer de la « promiscuité fonctionnelle » (Tobin 2010, p. 52) des protéines de ce genre, la conclusion selon laquelle des molécules dotées de fonctions différentes sont nécessairement des « espèces macromoléculaires distinctes » (Ibid.). Une espèce de molécules peut en effet remplir des fonctions différentes. Deuxièmement, le fait que les protéines de clair de lune peuvent avoir plusieurs fonctions ne réfute pas la thèse microstructuraliste selon laquelle leur profil fonctionnel est entièrement déterminé par leur structure microscopique.

17- D’autres exemples sont les ailes, les yeux, les opsines (Aizawa non publié) et la protéine kinase A dans le mécanisme de la consolidation de la mémoire (LTP) (Bickle 20o3, p. 144 ; Kistler 2004, p. 323 ; Aizawa et Gillett 2009, p. 198). 


\section{ESPÈCES NATURELLES, PROFIL CAUSAL ET CONSTITUTION MULTIPLE}

\section{5- La constitution multiple des espèces naturelles}

Le profil causal d'une espèce naturelle n'est que partiellement actualisé et partiellement connu. Jusqu'ici nous avons suggéré que les espèces naturelles sont postulées comme étant ce qui constitue la base commune d'un ensemble de dispositions indissociables. Certains auteurs ont suggéré une manière plus explicite et moins métaphysiquement obscure de caractériser l'identité des espèces naturelles. Hendry définit la doctrine microstructuraliste au sujet d'une espèce naturelle comme étant la " thèse selon laquelle ce sont des propriétés structurelles qui font de quelque chose un membre de cette espèce » (Hendry 2006, p. 865). Selon Hendry, cette thèse est plausible au moins pour les éléments chimiques mais aussi pour des substances complexes telles que l'eau.

Ne pourrions-nous pas identifier l'espèce naturelle sousjacente au profil causal d'une protéine simplement avec la structure microscopique de la protéine, c'est-à-dire avec l'ensemble de ses composantes atomiques et leur arrangement structurel ? Voici comment Tahko exprime cette hypothèse :

« (IDENT) Nécessairement, un échantillon d'une substance chimique A appartient à la même substance chimique que [l'échantillon] B si, et seulement si, A et B ont la même composition microstructurelle » (Tahko 2015, p. 804 $)^{18}$.

J'ai suggéré que les espèces naturelles sont postulées en sciences comme étant ce qui est sous-jacent à un ensemble de dispositions systématiquement associées. N'est-ce pas précisément la composition microstructurelle d'une molécule qui détermine l'ensemble de ses dispositions et fonctions?

Il ne fait aucun doute que le profil causal soit déterminé par la microstructure. Il s'agit là d'une conséquence du physicalisme et de la survenance des propriétés systémiques des objets complexes sur les propriétés de plus bas niveau de leurs composantes. Dans le cas des protéines, cela signifie que la structure tertiaire (et quaternaire) d'une protéine (et donc son profil causal, c'est-à-dire l'ensemble de ses dispositions) est une conséquence de sa structure primaire. Cependant, (IDENT) à la forme d'un biconditionnel. Or, seul l'un des deux conditionnels est une conséquence du physicalisme, à savoir : si les échantillons $\mathrm{A}$ et $\mathrm{B}$ de substances chimiques possèdent la même composition microstructurelle, alors $\mathrm{A}$ appartient à la même (espèce de) substance chimique que $\mathrm{B}$. En revanche, ce qui rend (IDENT) controversé est le second conditionnel selon lequel : si deux échantillons A et B appartiennent à la même substance chimique, alors $\mathrm{A}$ et $\mathrm{B}$ auront la même composition microstructurelle.

En d'autres termes, ce qui est controversé, c'est la question de savoir s'il existe une relation une à une entre une espèce naturelle, par exemple une espèce de protéine, identifiée par son profil causal (en partie actuel et en partie connu) et sa structure primaire, c'est-à-dire sa structure atomique.

Afin de comparer le niveau de la molécule entière avec le niveau de ses composantes, j'adopterai la convention d'appeler « macroscopique » le niveau de la molécule entière relativement au niveau de ses composantes atomiques. Il y a deux raisons de contester qu'il existe une correspondance une à une entre une propriété qui caractérise l'ensemble des composantes microscopiques - jointe aux relations structurelles entre ces composantes - et une propriété au niveau de la molécule entière sous-jacente au profil fonctionnel de la molécule.

Voici comment Tahko exprime la thèse selon laquelle il existe une telle relation une à une :

" (INST) Nécessairement, il y a une corrélation d'une à une entre (toutes) les propriétés chimiques d'une substance chimique et la microstructure de cette substance. » (Tahko 2015, p. 804)

Dans ce qui suit, je soutiendrai que (INST) n'est pas correct en général parce qu'il est possible que différentes microstructures donnent lieu à la même espèce naturelle macroscopique conçue en tant que ce qui est sous-jacent et unifie un ensemble de dispositions. Une macrostructure peut être déterminée par plusieurs microstructures.

Il est utile de démontrer d'abord que l'inverse n'est pas vrai. De prime abord, il pourrait sembler que la relation entre l'ensemble des composantes atomiques d'une molécule et son type chimique soit parfois une relation d'un à plusieurs, c'està-dire que la même structure microscopique puisse donner lieu à différents types de molécules. Cela pourrait sembler être le cas des isomères tels que le butane et l'isobutane dans la mesure où il s'agit là de différentes molécules qui ont exactement les mêmes composantes. Cependant, le cas des isomères montre simplement que la structure contient plus que l'ensemble des composantes. La structure contient également les relations entre les composantes. Or, si l'on inclut dans la structure, à la fois l'ensemble des composantes et l'ensemble des liaisons entre ces composantes, les isomères ne sont plus des contre-exemples à la thèse selon laquelle la structure microscopique d'une molécule détermine son type chimique.

Nous n'avons aucune raison de douter de la thèse physicaliste selon laquelle, si A et B sont deux échantillons qui ont la même composition microstructurelle alors l'échantillon A appartient à la même substance chimique que l'échantillon de B. Cependant, la thèse microstructuraliste est erronée parce que le second conditionnel qui compose le bicondition-

18 -(IDENT) peut être interprété soit en tant que thèse en métaphysique descriptive, soit en tant que thèse en métaphysique des sciences. Selon la première interprétation, la thèse exprime ce qu'est une espèce naturelle - ce qui détermine son identité - selon lïntuition du sens commun. Selon la seconde interprétation, en l'occurrence celle que nous adoptons ici, la thèse énonce ce que sont les espèces naturelles à la lumière de la science. 


\section{ESPÈCES NATURELLES, PROFIL CAUSAL ET CONSTITUTION MULTIPLE}

nel (INST) est faux. La multiconstituabilite (ou constituabilité multiple) montre qu'il n'est pas exact que si un échantillon A appartient à la même espèce de substance chimique qu'un échantillon B, A et B aient la même composition microstructurelle.

Si une substance chimique est multiconstituée, il existe deux ou plusieurs structures microscopiques qui donnent lieu à la même substance ${ }^{19}$. L'hémoglobine fournit une illustration d'une telle multiconstitution. Il existe une énorme variété de molécules d'hémoglobine même à l'intérieur de l'espèce humaine (Huisman, Carver et Efremov 1996). Néanmoins, seule une part de la diversité qui existe au niveau microstructurel aura des effets au niveau des dispositions chimiques et biologiques de l'hémoglobine. Ce qui fait d'une substance donnée de l'hémoglobine, c'est-à-dire ce qui est sous-jacent à son profil fonctionnel, est déterminé par une partie très spécifique de la structure microscopique. Seuls 9 des acides aminés composant la structure primaire des molécules d'hémoglobine sont présents dans toutes les molécules fonctionnelles d'hémoglobine. On les appelle les « résidus d'acides aminés hautement conservés dans les hémoglobines » (Anandhi 2014, p. 3-34). Des variations sur d'autres sites, à l'exclusion de ces 9 sites spéciaux, ne modifient pas le profil fonctionnel de la molécule (Ota, Isogai et Nishikawa 1997).

Pour des protéines telles que l'hémoglobine, il y a une relation de plusieurs à un entre leur microstructure (la séquence d'acides aminés qui constituent leur structure primaire) et les structures tertiaire et quaternaire qui déterminent leur profil causal. Cette multiconstituabilité réfute la thèse microstructuraliste selon laquelle il existe une relation d'un à un entre l'identité d'une substance chimique au niveau du type de la molécule entière et sa microstructure, c'est-à-dire l'ensemble de ses composantes et des relations entre celles-ci. Des structures microscopiques différentes peuvent donner lieu à un type unique de molécule, au sens où l'identité d'un type de molécules est déterminée par son profil causal.

La distinction entre les deux directions du biconditionnel qui définit le microstructuralisme nous permet de voir une faiblesse dans la défense du microstructuralisme par Goodwin. Le fait que « l'espace des conformations, et donc la capacité de réagir de différentes manières, soit déterminé par la microstructure » (Goodwin 2011, p. 540), correspond à la direction physicaliste du biconditionnel microstructuraliste. « L'espace de conformations » de Goodwin peut être interprété comme ce qui correspond à l'ensemble des dispositions, caractéristiques d'un type chimique donné, de manifester $\mathrm{M}_{\mathrm{i}}$ dans les circonstances $T_{i}$. Selon l'hypothèse défendue ici, cet ensemble de dispositions est déterminé par un pouvoir qui appartient au niveau de la molécule entière. Ce pouvoir macroscopique est lui-même déterminé par la microstructure, ce qui correspond à l'hypothèse physicaliste. Cependant, cela ne justifie pas « le monisme structurel à l'égard de la classification des protéines » (Goodwin 2011, p. 543) parce que cette doctrine affirme que l'autre direction du biconditionnel microstructuraliste est également correcte. Or, comme nous l'avons vu, la détermination du pouvoir macroscopique de la molécule (c'est-à-dire du pouvoir au niveau de la molécule entière) par la microstructure (c'est-à-dire l'ensemble de ses composantes et les relations entre celles-ci) est souvent une relation de plusieurs à un, au sens où plusieurs structures primaires différentes déterminent la même structure tertiaire (et éventuellement quaternaire) qui détermine à son tour l'ensemble des dispositions et des fonctions possibles qu'une protéine peut avoir.

\section{6 - Conclusion}

Les espèces naturelles sont des postulats théoriques qui fournissent un cadre à l'explication scientifique du fait que certaines propriétés et dispositions sont systématiquement associées. L’identité des espèces naturelles est déterminée par un ensemble de dispositions constituant son profil causal. Dans le cas des espèces biochimiques, certaines de leurs dispositions correspondent à des fonctions. Cette conception des espèces naturelles rend compte du fait qu'il arrive que certaines espèces biologiques occupent plus d'une fonction et que différentes espèces partagent une fonction. J'ai distingué deux thèses qui sont souvent identifiées avec la thèse microstructuraliste. Seule l'une d'elles est correcte. La partie correcte est la thèse physicaliste selon laquelle, si deux échantillons A et B partagent leur microstructure, alors A et $\mathrm{B}$ appartiennent à la même espèce chimique. La partie incorrecte est le conditionnel inverse. Il n'est pas correct de dire que si $\mathrm{A}$ et $\mathrm{B}$ appartiennent à la même espèce chimique, $\mathrm{A}$ et $\mathrm{B}$ partagent leurs microstructures. Des espèces biochimiques telles que les protéines sont « multiconstituées », au sens où

19 - La constitution multiple est analysée dans Aizawa et Gillett (2009). Lïdentification de la constitution multiple avec une forme de réalisation multiple peut être source de malentendus. Le désaccord entre les avocats de la constitution multiple et les avocats de ce que Gillett (2003) appelle la « conception plate » de la réalisation (Shapiro 2004) pourrait peut-être être surmonté en reconnaissant, avec ces derniers, que le terme « réalisation » désigne la relation entre un rôle et ce qui le remplit, en d'autres termes la relation entre un concept exprimé par un prédicat de premier ordre (qui décrit ce qui remplit le rôle) et un concept exprimé par un prédicat de second ordre (qui décrit le rôle). Dans le cas de l'hémoglobine (qui est un type de molécule qui remplit - ou « réalise »-le rôle de transporter l'oxygène dans le corps d'un certain nombre d'animaux), ce qui remplit le rôle est constitué de manière multiple : de nombreuses microstructures déterminent ce qui est la même espèce de substance remplissant le rôle, au sens de la conception des espèces naturelles défendue ici, en termes de profil fonctionnel. Il peut être source de malentendus d'appeler cette relation de constitution une forme de « réalisation ». On peut expliquer la source de tels malentendus en termes de distinction entre niveaux et ordres : les niveaux sont définis relativement à une hiérarchie de touts et de leurs parties. Un système en tant que tout et ses propriétés se situent à un niveau plus élevé que les parties de ce système et leurs propriétés. En revanche, les ordres correspondent à des niveaux de langage : un prédicat de second ordre est un prédicat défini à l'aide d'un quanteur existentiel qui porte sur des prédicats de premier ordre. La constitution est une relation entre des propriétés qui se situent à différents niveaux, à savoir entre les propriétés d'un objet complexe et les propriétés de ses parties, alors que le terme « réalisation » est plus naturellement utilisé pour désigner une relation entre des propriétés qui se situent au même niveau et qui correspondent à des prédicats d'ordres différents : un rôle fonctionnel est définipar un prédicat de second ordre; ce qui remplit ou « réalise » ce rôle est désigné par un prédicat de premier ordre. 


\section{ESPÈCES NATURELLES, PROFIL CAUSAL ET CONSTITUTION MULTIPLE}

des molécules avec des microstructures différentes peuvent appartenir à la même espèce chimique.

\section{Remerciements}

La recherche pour cet article a bénéficié du soutien financier du projet ANR-12-BSH3-0009 de l'ANR. Je remercie mes auditeurs au congrès de la SPS à Lille ainsi qu'A. Barberousse de leurs remarques critiques.

\section{RÉFÉRENCES}

ABBOTT, Barbara. 1997. A Note on the Nature of «Water». Mind, 106(422), 311-319. Article.

AHN, Woo-kyoung, KALISH, Charles, GELMAN, Susan A., MEDIN, Douglas L., LUHMANN, Christian, ATRAN, Scott, COLEY, John D., SHAFTO, Patrick. 2001. Why essences are essential in the psychology of concepts. Cognition, 82 (1), 59-69. Article.

AIZAWA, Ken. Compositional Realization. Article non publié.

AIZAWA, Ken, GILLETT, Carl. 2009. The (Multiple) Realization of Psychological and other properties in the Sciences. Mind and Language, 24, 181-208. Article.

ANANDHI, D. 2014. Introduction to Biochemistry and Metabolism, Noida (India) : Dorling Kindersley.

ARMSTRONG, David M. 1989. A Combinatorial Theory of Possibility. Cambridge : Cambridge University Press. Livre.

ARMSTRONG, David M. 2004. Truth and Truthmakers. Cambridge : Cambridge University Press. Livre.

BARBEROUSSE, Anouk, LUDWIG, Pascal. 2000. Les modèles comme fictions. Philosophie, 68, 16-43.

BICKLE, John. 2003. Philosophy and Neuroscience: A Ruthlessly Reductive Account. Dordrecht : Kluwer. Livre.

BIRD, Alexander. 2007. Nature's Metaphysics. Laws and Properties. Oxford : Clarendon Press. Livre.

BIRD, Alexander. 2015. The Metaphysics of Natural Kinds. Synthese, published online: 21 August 2015. Article.

BOYD, Richard. 1991. Realism, Anti-Foundationalism and the Enthusiasm for Natural Kinds. Philosophical Studies, 61, 127-148. Article.

BULLER, Andrew R., TOWNSEND, Craig A. 2013. Intrinsic evolutionary constraints on protease structure, enzyme acylation, and the identity of the catalytic triad. Proceedings of the National Academy of Sciences of the United States of America, 110 (8), E65361. Article.

CALLENDER, Craig. 2011. Philosophy of Science and Metaphysics. In FRENCH, Steven, SAATSI, Juha (eds.), Continuum Companion to the Philosophy of Science. London : Continuum. 33-54.

CHAKRAVARTTY, Anjan. 2007. A Metaphysics for Scientific Realism. Cambridge : Cambridge University Press. Livre.

CHURCHLAND, Paul M. 1979. Scientific Realism and the Plasticity of Mind. Cambridge : Cambridge University Press. Livre.

CHURCHLAND, Patricia S. 1986. Neurophilosophy: Toward a Unified Science of the Mind-Brain. Cambridge, MA: MIT Press. Traduit par M. Siksou. 1999. Neurophilosophie: l'esprit-cerveau. 1999. Paris : Presses Universitaires de France.

COVA, Florian. 2011. Qu'en pensez-vous ? Une introduction à la philosophie expérimentale. Paris : Germina.

COVA, Florian. 2012. Qu'est-ce que la philosophie expérimentale ? In COVA, Florian, DUTANT, Julien, MACHERY, Edouard, KNOBE, Joshua, NICHOLS, Shaun, NAHMIAS, Eddy (ed.), La Philosophie Expérimentale. Paris : Vuibert. 5-29.

DE JONG, Wilfried W., LEUNISSEN, Jack A., VOORTER CHRISTINA, E.M. 1993. Evolution of the alpha-crystallin/small heatshock protein family. Molecular Biology and Evolution, 10(1), 103-26. 
REVUE

DE LA SOCIÉTÉ

DE PHILOSOPHIE

DES SCIENCES

\section{ESPÈCES NATURELLES, \\ PROFIL CAUSAL ET \\ CONSTITUTION MULTIPLE}

DUMSDAY, Travis. 2013. Using Natural-Kind Essentialism to Defend Dispositionalism. Erkenntnis, 78, 869-880. Article.

FODOR, Jerry A. 1974. Special Sciences, or the Disunity of Science as a Working Hypothesis. In BLOCK, Ned. 1981. Readings in the Philosophy of Psychology, vol. 1; et In FODOR, Jerry A. 1981. Representations. Cambridge, MA: MIT Press. Chapitre.

GELMAN, Susan. 2003. The Essential Child: Origins of Essentialism in Everyday Thought. New York : Oxford University Press. Livre.

GILLETT, Carl. 2003. The Metaphysics of Realization, Multiple Realizability, and the Special Sciences. Journal of Philosophy, 100, 591-603.

GOODWIN, William. 2011. Structure, function, and protein taxonomy. Biology and Philosophy, 26, 533-545. Article.

HEMPEL, Carl G. 1965. Empiricist Criteria of Cognitive Significance. Aspects of Scientific Explanation. New York : Free Press. 101-122.

HENDRY, Robin F. 2006. Elements, Compounds and other chemical kinds. Philosophy of science, 73, 864-875. Article.

HUISMAN, Titus H.J., CARVER, Marianne F.H., EFREMOV, Georgi D. 1996. A Syllabus of Human Hemoglobin Variants. Augusta, GA : The Sickle Cell Anemia Foundation.

JACKSON, Frank. 1998. From Metaphysics to Ethics. Oxford : Clarendon Press. Livre.

KIM, Jaegwon. 1998. Mind in a Physical World. Cambridge, MA : MIT Press. Traduit par F. Athané et E. Guinet. 2014. L'esprit dans un monde physique. Paris : Ithaque.

KISTLER, Max. 2002. The Causal Criterion of Reality and the Necessity of Laws of Nature. Metaphysica, 3, 57-86.

KISTLER, Max. 2004. Matérialisme et réduction de l'esprit. In DUBESSY, Jean, LECOINTRE, Guillaume, SILBERSTEIN, Marc (eds.). Les matérialismes (et leurs détracteurs). Paris: Syllepse, 309-339. Repris In SILBERSTEIN, Marc (ed.). 2013. Matériaux philosophiques et pour un matérialisme contemporain: Sciences, ontologie, épistémologie. Paris : Editions Matériologiques. 919954.

KISTLER, Max. 2007. La réduction, l'émergence, l'unité de la science et les niveaux de réalité. Matière Première. 2, 67-97. Repris In SILBERSTEIN, Marc (ed.). 2013. Matériaux philosophiques et pour un matérialisme contemporain: Sciences, ontologie, épistémologie. Paris : Editions Matériologiques. 179-212.

KISTLER, Max. 2012. Powerful Properties and the Causal Basis of Dispositions. In BIRD, Alexander, ELLIS, Brian, and SANKEY, Howard (ed.), Properties, Powers and Structures. Issues in the Metaphysics of Realism. New York and Oxford : Routledge. 119137.

KISTLER, Max. À paraître, a. L'esprit matériel : réduction et émergence. Paris : Ithaque.

KISTLER, Max. À paraître, b. Natürliche Eigenschaften und natürliche Arten. In SCHRENK, Markus (ed.). Handbuch Metaphysik. Stuttgart/Weimar : J.B. Metzler.

LEWIS, David. 1986. On the Plurality of Worlds. Oxford : Blackwell.

LADYMAN, James, ROSS, Don, SPURRETT, David, COLLIER, John. 2007. Every Thing Must Go: Metaphysics Naturalized.
Oxford : Oxford University Press. Livre.

LOWE, Edward Jonathan. 2001. The Possibility of Metaphysics. Oxford: Clarendon Press. Livre.

MALATERRE, Christophe. 2010. Les origines de la vie. Emergence ou explication reductive. Paris : Hermann.

MANAFU, Alex. 2014. A Novel Approach to Emergence in Chemistry. In SCERRI, Eric, and MCINTYRE, Lee, (ed.). Philosophy of Chemistry. Growth of a New Discipline. Dordrecht : Springer. Chapitre.

MANAFU, Alex. Microstructuralism: An Analysis. Non publié. MAUDLIN, Tim. 2007. The Metaphysics Within Physics. New York: Oxford University Press. Livre.

MELLOR, David Hugh 1991. Properties and Predicates. In Matters of Metaphysics. Cambridge : Cambridge University Press. 170-82. MELLOR, David Hugh. 2012. Nature's Joints: A Realistic Defense of Natural Properties. Ratio (new series), 25, 387-404. Article.

MILL, John Stuart. 1843. A System of Logic, Ratiocinative and Inductive. 2002. Reprise de l'édition de 1891. Honolulu (Hawaii) : University Press of the Pacific. Livre.

MILLS, Eugene. 2014. Mellor on the Sparseness of Natural Properties. Ratio (new series), 27, 350-355. Article.

MORRIS, Catherine, LECAR, Harold. 1981. Voltage Oscillations in the Barnacle Giant Muscle Fiber. Biophysical Journal, 35, 193-213. Article.

OKASHA, Samir. 2002. Darwinian Metaphysics: Species and the Question of Essentialism. Synthese, 131, 191-213. Article.

OTA Motonori, ISOGAI, Yasuhiro, NISHIKAWA, Ken (1997) Structural requirement of highly-conserved residues in globins. FEBS Letters, 415 (2), 129-133. Article.

PRIOR, Elizabeth W., PARGETTER, Robert, JACKSON, Frank. 1982. Three Theses about Dispositions. American Philosophical Quarterly, 19, 251-257.

QUINE, Willard Van Orman. 1939a. A Logistical Approach to the Ontological Problem. In The Ways of Paradox and Other Essays, Revised edition, Cambridge, MA : Harvard University Press. 1976. 197-202.

QUINE, Willard Van Orman. 1939b. Designation and Existence. Journal of Philosophy, 36, 701-709. Article.

QUINE, Willard Van Orman. 1948. On What There Is. In From A Logical Point of View. Second edition. Cambridge, MA : Harvard University Press, 1980.

SAIBIL, Helen. 2013. Chaperone machines for protein folding, unfolding and disaggregation. Nature Reviews. Molecular Cell Biology, 14(10), 630-42. Article.

SETOH, Peipei, WU, Di, BAILLARGEON, Renée, GELMAN, Rochel. 2013. Young infants have biological expectations about animals. Proceedings of the National Academy of Science USA, 110, 15937-15942. Article.

SHAPIRO, Larry A. 2004. The Mind Incarnate. Cambridge, MA : MIT Press.

SHOEMAKER, Sydney. 1980. Causality and properties. In Identity, Cause and Mind. Cambridge : Cambridge University Press, p. 206-233. Repris In MELLOR, David Hugh, OLIVER, Alex (ed.). 1997. Properties. Oxford : Oxford University Press. 228-254. Chapitre. 
SLATER, Matthew H. 2015. Natural Kindness. British Journal for the Philosophy of Science, 66(2) : 375-411. Article.

SOUSA, Paolo, ATRAN Scott, MEDIN, Douglas. 2002. Essentialism and Folkbiology: Evidence from Brazil. Journal of Cognition and Culture, 2, 195-223. Article.

STRAWSON, Peter Frederick. 1959. Individuals. Traduit par A. Shalom et P. Drong. 1973. Les Individus. Essai de métaphysique descriptive. Paris : Seuil. Livre original.

TAHKO, Tuomas. 2015. Natural Kind Essentialism Revisited. Mind, 124(495), 795-822. Article.

TOBIN, Emma. 2010. Microstructuralism and macromolecules: the case of moonlighting proteins. Foundations of Chemistry, 12, 41-54. Article.

TOBIN, Emma. 2013. Are natural kinds and natural properties distinct?. In MUMFORD, Stephen, TUGBY, Matt (eds.). Metaphysics and Science. Oxford : Oxford University Press. 164-182. Chapitre. TOMPA, Peter, SZASZ, Csilla, BUDAY, Laszlo. 2005. Structural disorder throws new light on Moonlighting. Trends in Biochemical Sciences, 30, 484-9. Article.

VAN HOLDE, Kensal E., MILLER Karen I. 1995. Hemocyanins. Advances in Protein Chemistry, 47, 1-81. Article.

VORMS, Marion. 2011. Qu'est-ce qu'une théorie scientifique ? Paris : Vuibert.

\section{HISTORIQUE}

Ce texte est la version modifiée de l'exposé prononcé le 26 juin 2014, durant le débat plénier, au Ve Congrès de la Société de philosophie des sciences (« Métaphysique des sciences », Lille, 25-27 juin 2014).

\section{SITE WEB DE LA REVUE}

sites.uclouvain.be/latosensu/index.php/latosensu/index

ISSN 2295-8029

DOI http://dx.doi.org/10.20416/lsrsps.v3i1.573

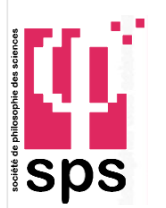

SOCIÉTÉ DE PHILOSOPHIE DES SCIENCES (SPS) École normale supérieure

45, rue d'Ulm 75005 Paris

www.sps-philoscience.org
CONTACT ET COORDONNÉES :

Max Kistler

Université Paris 1 Panthéon Sorbonne IHPST (Institut d'Histoire et de Philosophie des Sciences et des Techniques)

13 rue du Four

75006 Paris

mkistler@univ-paris1.fr 